\title{
ENVIRONMENT SENSOR FOR HOME SLEEP MONITORING
}

\author{
László Árvai \\ Department of System Development \\ Bay Zoltán Nonprofit Ltd. for Applied Research \\ 3519 Miskolc, Iglói u. 2., e-mail: laszlo.arvai@bayzoltan.hu \\ Dezső Vass \\ Department of System Design \\ Bay Zoltán Nonprofit Ltd. for Applied Research \\ 3519 Miskolc, Iglói u. 2., e-mail: dezso.vass@bayzoltan.hu \\ András Leleszi \\ Department of System Design \\ Bay Zoltán Nonprofit Ltd. for Applied Research \\ 3519 Miskolc, Iglói u. 2., e-mail: leleszi.andras@bayzoltan.hu
}

\begin{abstract}
The sleep is a very important period of everyone's life cycle. It has importance in the regeneration and preparation for the next day's activity. Therefore, the quality of the sleep is important the effectiveness of the next day or long-term ineffective sleep will degrade the concentration of the person which can be serious problem in certain professions like bus drivers, pilots. The objective measurement, monitoring and evaluation of the sleep quality is important either in the sleep laboratory or even in the patient home. There are several type of sleep sensors, based on different working principle, contact, contactless and indirect. They provide important information about the human condition while asleep. However, it turned out the environment monitoring also provides valuable information about the sleep quality. Especially in the case of home sleep monitoring the environment monitoring is essential. This article explains the home sleep monitoring and introduces an environment monitoring device developed for home sleep monitoring.
\end{abstract}

Keywords: sleep sensors, home sleep monitoring, environment monitoring, air quality monitoring

\section{Introduction}

The term insomnia is very often appearing in the medical literature or even in our daily life. Since the large percentage of the population has sleeping difficulties and in certain jobs like drivers, pilots the insomnia might have serious consequences the measurement of sleep quality gaining more interest.

Different sources give slightly different percentage about the involvement of the population in insomnia, but the commonly accepted value seems to be at least $30 \%$ of the population [1][2]. As the $30 \%$ of the population suffers from at least one of the insomnia types, $10-15 \%$ has chronic, an additional 25$35 \%$ from transient or occasional insomnia. Estimates vary since the definitions and the diagnostic criteria is not exact. There are several types of sleep disorders as listed in DSM-5 [3]:

- Insomnia

- Sleep Apnea

- Restless Legs Syndrome 
- Narcolepsy

- Hypersomnolence (hypersomnia) Symptoms

- Circadian Rhythm Sleep Disorder

- REM Sleep Behavior Disorder

The effect of disorders can be

- Fatigue or low energy

- Daytime sleepiness

- Cognitive impairments (e.g., attention, concentration, memory, learning)

- Mood disturbance (e.g., irritability, dysphoria, anxiety)

- Behavioral problems (e.g., hyperactivity, impulsivity, aggression)

- Impaired academic or occupational function

- Impaired interpersonal/social functioning

In severe cases it may cause dangerous situations while driving vehicles especially heavy vehicles. The European Union directive 2014/85/EU requires drivers with moderate or severe apnea to refer to authorized medical advice and after identifying apnea receive CPAP treatment and prove that their vigilance is regained before their license is issued [4].

To achieve a diagnosis on sleep apnea there are several methods applied in multiple stages. The first phase is a pre-screening asking the patient to fill-in a questionnaire aiming to acquire information on one's symptoms, senses like snoring or witness (bed partner) experience during the sleep cycle.

The most widespread questionnaires are

- Epworth Sleepiness Scale (ESS)

- Berling Questionnaire (BQ)

- STOP BANG Questionnaire (SBQ)

The result coming from questionnaires are subjective so they are used only in the pre-screening phase of apnea detection.

According to a clinical validation study the efficiency of the questionnaires varies from $40 \%$ up to 95-100\%. Obviously higher efficiency can be achieved in more severe cases. The apnea related sleep deprivation can root in different problems, anatomic lesions (e.g. atherosclerotic [5], lingual tonsil hypertrophy [6])), airway obstacles, psychiatric disturbances. When the doctor decides to order sleep laboratory examination the patient's sleep cycle is observed during one night. The gold standard for the observation is polysomnography (PSG) which records the patient's vital parameters throughout the sleep phases. Main fields of the recorded parameters are brain activity (EEG), cardiac (blood pressure, EKG), oximetry (SPO2) eye movement (EOG), snoring and limbic movements. This is the most precise and trustful method. The results can be slightly affected by the so called First Night Effect (FNE). FNE is the alteration of sleep architecture observed on the first night of polysomnographic (PSG) studies. It is problematic to identify whether the FNE reflects adaptation to the sleeping environment, the equipment or both of them [7]. In addition, there are some practical and economic considerations with this way of diagnosis. In Hungary there are only 12 sleep laboratories operating and 20 certified somnologist resulting long queues for the diagnosis. Besides that, there are some disabled and elderly people not mobile enough to visit a sleep lab. 


\section{Sleep Monitoring at Home}

The sleep monitoring is usually done in a certified sleep laboratory. However, in the recent years home monitoring methods and solutions have been developed and validated in clinical studies. The economic efficiency of the home monitoring was also subject of studies [8] and the conclusion was that it is effective and can be recommended to be used in the pre-screening phase. The solutions of home monitoring vary from a simple smart phone application which records snoring sounds and vibration to the multiple sensor solution attached to the patient's body during the bedtime. The most preferred solutions for the patients are non-contact solutions however it might decrease the overall precision of the measurement.

The sleeping monitoring applications usually uses a score-based calculation (1). The score is computed from several factors and provides an overall sleep quality measure. The usual components of the sleep score are:

- Total Sleep Time

- Sleep Goal

- Deep Sleep Percentage

- Total Number of Awakenings

- Sleep Latency (when sleep is recorded manually)

- Total Awake Time (between sleep wake-up and onset time)

- Average HR during the sleep duration.

- Arm \& Leg Disturbance

- Snoring Disturbance

- Number of Low SPO2 readings during the sleep

- Sleep Goal Achieved = Percentage of Total Sleep versus the Sleep Goal

$$
\text { SleepEfficiency }=\frac{\text { TotalSleepTime }}{\text { TotalTimeinBed }}
$$

Sleep Score Penalties (2) - listed below (examples, not complete):

- Latency Penalty - Penalty is computed when latency is greater than 20 minutes.

- Awakenings Penalties - Penalty is computed when total number of awakenings are greater than certain number.

- Deep Sleep Penalty - Penalty is computed when Deep Sleep percentage is less than $35 \%$.

- Arm \& Leg Disturbance Penalty - Penalty is computed based upon number of minutes of mild, moderate, and several movements.

$$
\text { Sleep score }=(100-\text { penalty points }) * \text { Sleep goal Achieved } * \text { Sleep Efficiency }
$$

\section{Home Sleep Monitoring}

The sleep monitoring at home is getting more importance since the potentially endangered patients or the persons who requires periodical sleep test because of their profession can get tests more easy and economical way.

It is also providing some solution for the "First Night Effect" since at home it happens rarely. With the home monitoring it is also possible to provide the required periodicity of the sleet monitoring examination, in practice the continuous monitoring is also possible. 
Since the cost of the home sleep monitoring system must be reasonable, the high precision clinical devices cannot be afforded. The home monitoring devices provides less accurate result than their clinical counterpart, however they still can be very useful for the prescreening phase of the sleep monitoring.

Because of the mentioned reasons only a few types of sleep monitoring device can be used at home.

\subsection{Monitoring with Bed Sensor (Under-Mattress Sensor)}

Generally, a thin, narrow, flexible strip is used as sensor which it is placed under the mattress or possibly a sheet. During operation, the sensor not only senses the normal movement of the sleeping person, but also detects micro-movements of the body (for example: heart contractions).

This solution includes a mobile application and a portal besides a bed sensor. Raw and derived data (scoring) are displayed on the portal. The portal provides an opportunity to display trends and it can be explaining the interpretation of individual sleep characteristics to non-clinical persons. This advantage of the solution is that its continuous use is almost invisible to the person and they cannot forget to use it day by day. Using it after installation does not require user intervention. The sensor requires external power supply and therefore the wiring must be carefully installed because it must be ensured that the sleeping person cannot be move the sensor and cables and accidently cannot switch off the device.

\subsection{Monitoring with IR-UWB Radar}

The frequency interval of the IR-UWB radar is 3-10 GHz. This frequency spectrum provides lower energy consumption than the Doppler radar and this frequency interval provides less interference with other devices. The UWB device should be close to sleeping person (about $1 \mathrm{~m}$ ) that it is able to detect the breathing, heart-rate and other movement.

The recorded vital parameters partly covering the PSG recorded parameters, e.g. breathing rate (BR), heart rate (HR) and movement. It also can detect sleep apnea events.

\section{Environment Monitoring}

The environment monitoring plays almost same important role in the sleep monitoring as the monitoring of the patient physical parameters. This is especially true when the patient is monitored not in the hospital in a well-controlled and stable environment but in her/his home where the environment might vary and the sleeping quality might affect heavily by the environment condition. Therefore, for proper home sleep quality monitoring an environment monitoring device also requires.

\subsection{Parameters to be Monitored}

There are several environment parameters to affect sleep quality. The most obvious parameters which monitored by almost all environment monitoring device are the temperature, humidity. The sleep quality also might affected by the noise and lighting conditions of the bedroom [10].

The air quality also plays an important role in the environment monitoring even it is not so often monitored. The $\mathrm{CO}_{2}$ monitoring [11] and dust concentration is an important indication of the ventilation and also affects the air quality and hence the sleep quality directly.

\subsection{Home Environment Monitoring Device}

The home environment monitoring device must fulfil several expectations. The device itself is only responsible for the data collection but the data storage must be done in a cloud service or a centralized 
server computer. Since the health monitoring data also stored on this server it makes the data processing, analysis easier since the complete dataset (health, environment) is available in one system. Therefore, the device needs to have a communication interface which is according the recent trends must be a wireless communication.

The low power consumption is also a requirement since the device is not just can be operated from external power supply, but it also must be able to run from its internal batteries for long period and also must be able to manage the battery, charge, estimate the remaining charge in the battery and issue alarm condition when battery is low.

The architecture of the system is shown on Figure 1. The main module is containing a CPU controlling the operation of the sensor system. The microcontroller is based on ARM core, has several peripheral components and has very low power consumption. It is normally in deep sleep mode and wakes up periodically to read the sensor values and transfer it using the communication interfaces.

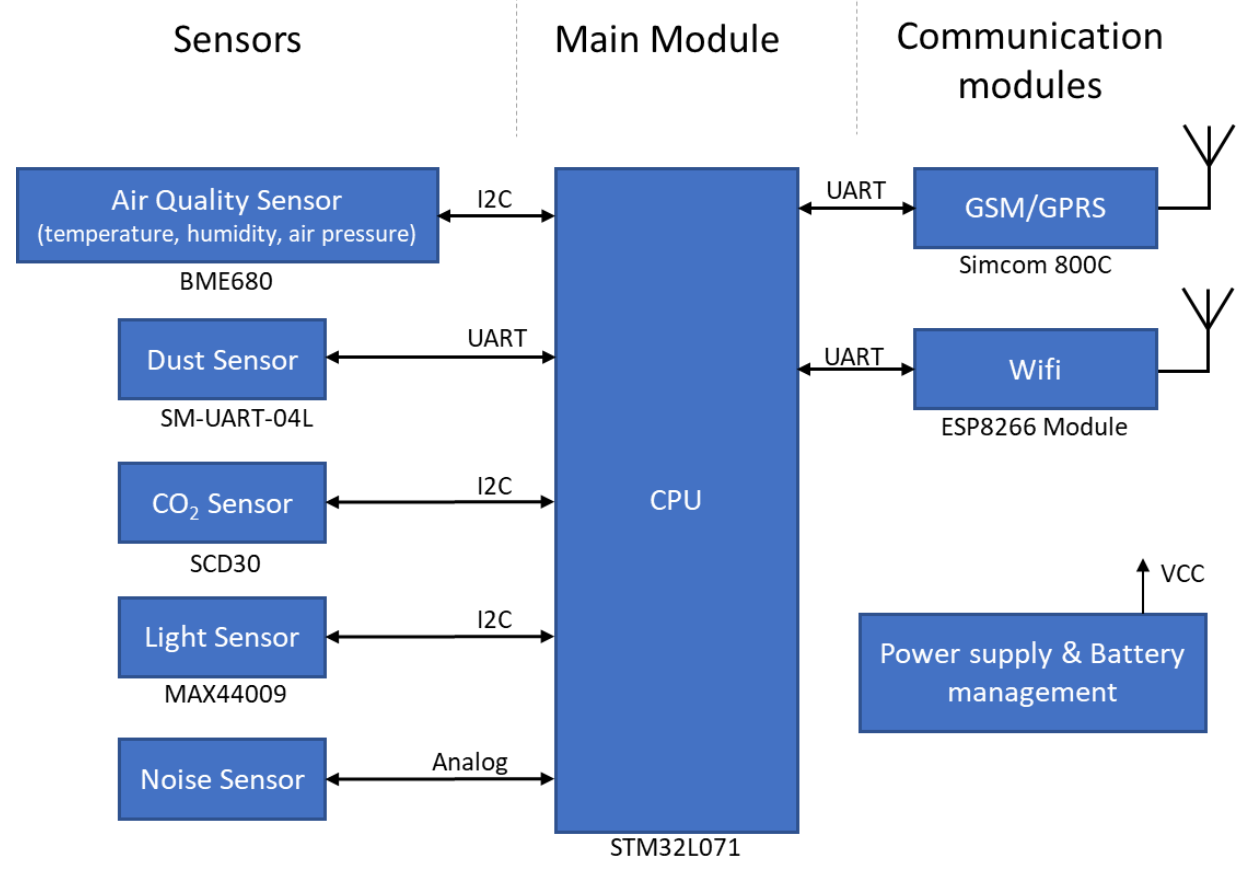

Figure 1. System architecture

The communication interface consists two modules. It is capable of communicating over standard GSM network or where the Wi-Fi connection is available it can be used since the sensor has Wi-Fi module.

The system can be powered either by the external power source or internal battery. The battery also managed by the system in the presence of external power it can charge the battery, measure its charge state and can provide warning message when the battery charge is low.

The sensors are the most important components of the system. On order to provide flexibility in terms of configuration the sensors are connected using a proprietary bus system which can accommodate six sensor modules with various interfaces. The sensors are chosen for this application are the air quality, dust, $\mathrm{CO}_{2}$, Light and Noise sensors. 
The air quality sensor is a Bosch BME680 type sensor. It can measure the temperature, air pressure and humidity. This sensor provides general information about the environment condition and the temperature alone is a very important parameter of the sleep quality.

The dust sensor can measure dust particle concentration with particle size of $2 \sim 6 \mu \mathrm{m}$. The type of this sensor is Amphenol SM-UART-04L. This measurement provides information about the cleanness of the air and also about the ventilation frequency.

The $\mathrm{CO}_{2}$ sensor is Sensirion SCD30 capable of measuring $\mathrm{CO}_{2}$ concentration which also provides information about the ventilation of the room and the $\mathrm{CO}_{2}$ concentration directly affect the sleeping quality as well.

The light sensor is a MAX44009 type sensor capable of measuring very dark light conditions from 0.045 lux with a very wide dynamic range of 22-bit. The light also affects the sleeping quality and could be a very important parameter for people who's working hour mostly includes night therefore it is often requires for them to sleep at daytime.

The noise sensor is basically a microphone with an amplifier, an A-Weighting filter and a peak rectifier providing analog signal sampled by the A/D converter of the microcontroller. The noise is also a very important parameter of the sleeping quality since the high peak level of noise significantly might reduce the quality of sleeping.

The completed device is shown on Figure 2. The left side of the circuit board contains the microcontroller, communication modules and power supply, while the right side of the image shows the sensor modules.

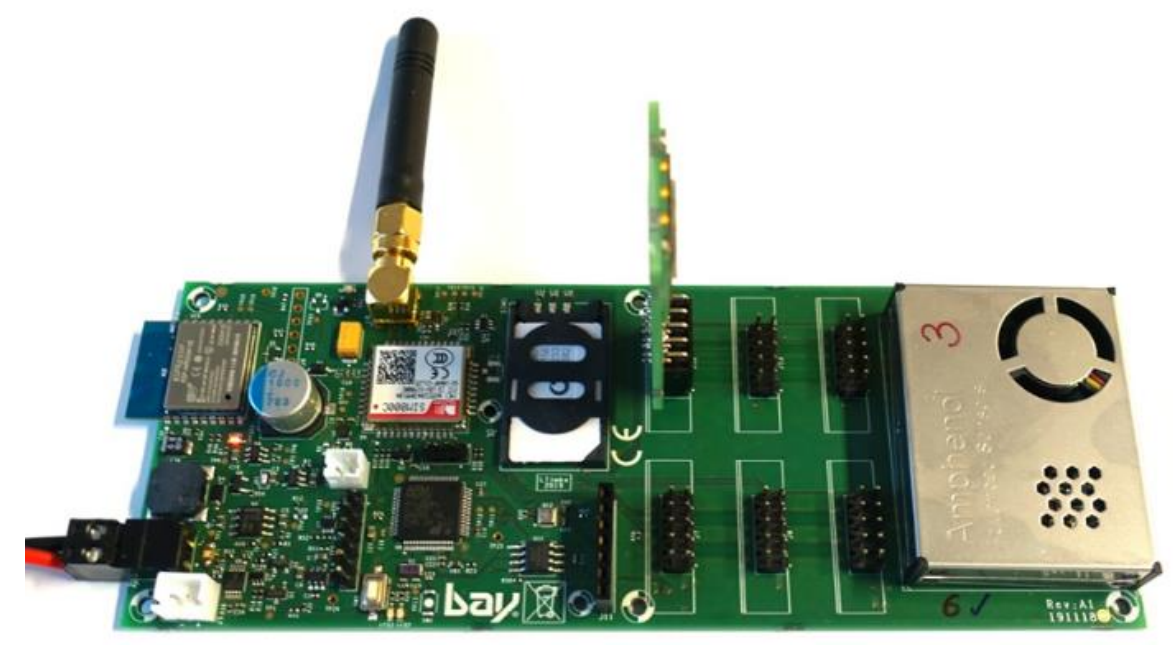

Figure 2. Environment monitoring device without case

The sensor modules can be changed, the system always can be configured for the specific task.

\section{Evaluation}

The location of the environmental sensors was at center of the bedroom and at a height of about 80-100 $\mathrm{cm}$. In the pilot experiment the bedroom air temperatures averaged at $20.2^{\circ} \mathrm{C}$ with the window closed and at $17.2^{\circ} \mathrm{C}$ when it was open (the pilot was in winter time, so window was rarely opened, it was opened only for short period of the ventilation). The humidity was $40 \%$ and $\mathrm{Sd}$ was 7.8 in average. The 
range of carbon dioxide concentration were between 1610 (after 1 hours from falling asleep) and 3250 ppm when the windows was closed for long time.

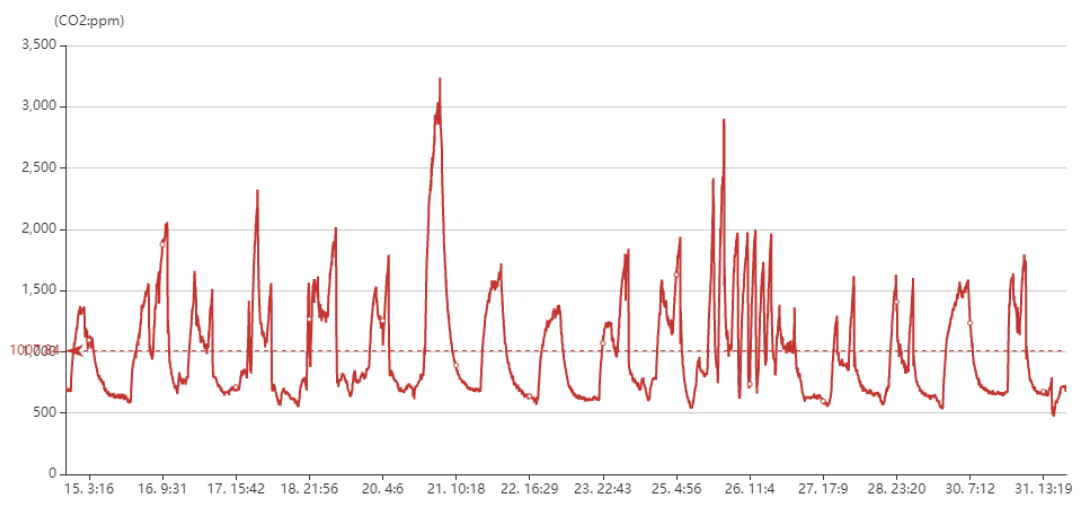

Figure 3. Carbon dioxide concentration vs. time

Concentration changes can also be inferred for night door openings. (if the bedroom door is closed during sleep)

\section{Conclusions}

The interest for the sleep quality assessment increased in the recent years since the bad sleeping quality affect the person's life, efficiency in work and the insomnia might cause dangerous situation for the drivers, pilots.

Therefore, the sleep monitoring gaining more importance, however the capacity of the polysomnography laboratory is limited and the First Night Effect might distort the measurement result. For these reasons the home sleep monitoring getting more and more importance. There are some sleep monitoring devices for health-related monitoring but especially at home the sleeping environment also an important factor and needs to be monitored. In this paper we have presented a low cost, versatile home environment monitoring device capable of measure several important environmental parameters and collect them on a server computer. This makes possible the better analysis of the sleep quality and better understanding the reasons behind the low sleep quality.

This device also can be used without the sleep monitoring devices, in this case it still can provide important information about the sleeping environment and can help improving it by changing the bedroom temperature, more frequent ventilation or simply changing the light conditions.

The future development plan includes to add gateway functionality so the health measurement devices could directly communicate with the gateway and the gateway would be responsible for sending their data to the server.

\section{References}

[1] Thomas, R.: Insomnia: definition, prevalence, etiology, and consequences, Journal of clinical sleep medicine: JCSM: official publication of the American Academy of Sleep Medicine vol. 3,5 Supply, (2007) pp. 7-17.

[2] National health Service, Sleep problems in the UK highlighted, 2011, https://www.nhs.uk/news/lifestyle-and-exercise/sleep-problems-in-the-uk-highlighted/ 
[3] Espie, C., Kyle, S. D., Hames, P., Cyhlarova, E., Benzeval, M.: The daytime impact of DSM-5 insomnia disorder: comparative analysis of insomnia subtypes from the Great British Sleep Survey, The Journal of Clinical Psychiatry 2012 Dec, pp. 1478-1484.

https://doi.org/10.4088/jcp.12m07954

[4] Ghosh, D., Mackay, T. W., Riha, R. L.: European Union directive 2014/85/EU on driver licensing in obstructive sleep apnoea: early experiences with its application in the UK, Breathe (Sheff) (2016), pp. 59-63. https://doi.org/10.1183/20734735.008016

[5] Dempsey, J. A., Veasey, S. C., Morgan, B. J., O'Donnell, C. P.: Pathophysiology of sleep apnea, 2010, Physiological Reviews 2010, pp. 47-112. https://doi.org/10.1152/physrev.00043.2008

[6] Dündar, A., Özünlü, A., Sahan, M., Özgen, F.: Lingual tonsil hypertrophy producing obstructive sleep apnea, The Laryngoscope vol. 106, issue 9, (1996) pp. 1167-1169. https://doi.org/10.1097/00005537-199609000-00022

[7] Herbst, E., Metzler, T. J., Leonici, M., McCaslin, S. E., Inslicht, S., Marmar, C. R., Neylan, T. C.: Adaptation effects to sleep studies in participants with and without chronic posttraumatic stress disorder, Psychophysiology vol. 47(6), (2010) pp. 1127-33. https://doi.org/10.1111/j.1469-8986.2010.01030.x

[8] Sherrill, W. C.: Home sleep testing, 2013, https://www.acc.org/latest-in-cardiology/articles/2014/07/18/15/42/home-sleep-testing

[9] Kim, R. D., Kapur, V. K., Redline-Bruch, J., Rueschman, M., Auckley, D. H., Benca, R. M., Foldvary-Scaher, N. R., Iber, C., Zee, P. C., Rosen, C. L., Redline, S., Ramsey, S. D.: An economic evaluation of home versus laboratory-based diagnosis of obstructive sleep apnea, Sleep vol. 38, (2015) pp. 1027-1037. https://doi.org/10.5665/sleep.4804

[10] Lobato, F., Silva, B., Bem, R. D., Miranda, D.: Non-invasive sleep-environment monitoring system, 2015 Proceedings of the 8th ACM International Conference on PErvasive Technologies Related to Assistive Environments https://doi.org/10.1145/2769493.2769563

[11] Strøm-Tejsen, P., Zukowska, D., Wargocki, P., Wyon, D. P.: The effects of bedroom air quality on sleep and next-day performance, 2016, Indoor Air. 2016 Oct, pp. 679-686. https://doi.org/10.1111/ina.12254 